mans, die eine Interdependenz von Bildnis und Sprache, von ästhetischen und kulturellen Fremdheitserfahrungen vermittelt. Auch das Drama Andorra (1961) zeigt den Zusammenhang zwischen Sprache, Bildnis und Identität sowie die fatalen Konsequenzen der antisemitischen Bildnismacherei auf. In der Umdichtung des Schweizer Gründungsmythos in Wilhelm Tell für die Schule (1970) verschränken sich die Fremdheitserfahrungen des Protagonisten mit der parodistisch verfremdeten Rhetorik nationaler Identitätsbildung: Die Relevanz der Intertexte und die rhetorischen Verfahren der Satire gehören aber auch zu jenen Aspekten der Fremdheit, welche die Wechselwirkung ästhetischer und kultureller Alteritätsphänomene illustrieren.

Alle im Band besprochenen Texte verfügen auch über eine gedächtnistheoretische Relevanz. Der ägyptische Heinrich und Im Kongo leisten einen Beitrag zur Diskussion um die Schweizer Vergangenheitsbewältigung und die Migrationsromane, die ähnlich wie Nadj-Abonjis interkultureller Familienroman Tauben fliegen auf grenzüberschreitende
Bewegungen zwischen Ostmitteleuropa und dem Westen beschreiben, gewähren einen Einblick in die Verschränkungen von Erinnerungskultur, Geschichtspolitik und kulturellem Gedächtnis in westlichen und in postkommunistischen Gesellschaften. In diesem Kontext ließen sich weitere Grenzgängergeschichten (z.B. von deutsch-ungarischen Autorinnen) auf Verflechtungen zwischen Schreibstrategien und migratorischen Identitätsmustern hin untersuchen.

Ein Verdienst des Bandes ist es, dass er nebst der Neueinordnung bzw. Neuinterpretation des Schaffens Deutschschweizer Autorinnen und Autoren die Relevanz der Ansätze aus der Xenologie für die Literaturanalyse beleuchtet. Damit erweisen sich die Literarischen Grenzgänge als eine anregende Lektüre nicht nur für Germanisten bzw. Erforscherinnen der Schweizer Literatur und Kultur, sondern auch für alle, die aktuelle Fragen der Erinnerungskultur und Geschichtspolitik in einer transnationalen Perspektive verstehen möchten.

Anett Szegvölgyi-Pócsik

\title{
Birgit Huemer, Eve Lejot, Katrien L. B. Deroey (Hg.): Academic writing across languages: multilingual and contrastive approaches in higher education. L'écriture académique à travers les langues: approches multilingues et contrastives dans l'enseignement supérieur. Wissenschaftliches Schreiben sprachübergreifend: mehrsprachige und kontrastive Ansätze in der Hochschulbildung
}

Wien: Böhlau Verlag 2019 - ISBN: 978-3-205-20705-4 - 37,99€

In dem Sammelband werden Ansätze zum wissenschaftlichen Schreiben in verschiedenen Sprachen als wichtige Aspekte von Hochschulbildung disku- tiert. Das Buch ist aus dem gleichnamigen Symposium hervorgegangen, das 2016 an der Universität Luxemburg stattfand. Gleichzeitig handelt 
es sich dabei um den ersten Band der neu gegründeten Reihe Schreibwissenschaft, die von der Gesellschaft für wissenschaftliches Schreiben (GewissS) herausgegeben wird.

Der Sammelband enthält zwölf Beiträge in drei Sprachen (Deutsch, Englisch, Französisch), die in folgende vier Teile gegliedert sind: didaktische Herangehensweisen (>Teaching approaches $<$, sprachliche Merkmale (>Linguistic features $<$ ), Schreibpraktiken (>Writing practices $<$ ) und eine $\mathrm{Zu}$ sammenführung der Ergebnisse sowohl des Symposiums als auch der Beiträge des Sammelbands.

$\mathrm{Hu}$ eröffnet das Vorwort mit der Aussage, dass Wissenschaft global betrachtet schon immer mehrsprachig war. Sie beschreibt den wachsenden Druck und die rasanten Veränderungen, denen die heutigen Hochschulen als Orte der Forschung und der Lehre unterliegen. Dabei geht sie auch auf die Rolle des Englischen als Lingua franca ein und schließt damit, dass wissenschaftliches Schreiben »hochgradig komplex, normativ und politisch« (11) ist.

Huemer gibt in der Einleitung einen knappen, jedoch umfassenden Überblick über die Forschungsentwicklung, die den Beiträgen des Sammelbands zugrunde liegt. Sie stellt Untersuchungen der kontrastiven und interkulturellen Rhetorik vor und benennt sprachvergleichende Studien. Dabei unterteilt sie Letztere in drei Gruppen: 1. Studien, die englische Texte von SchreiberInnen mit Englisch als Erstsprache und SchreiberInnen mit Englisch als Fremd- oder Zweitsprache vergleichend untersuchen, 2 . sprachvergleichende Studien, bei denen eine der untersuchten Sprachen nicht Englisch ist, und 3. sprachvergleichende Studien, bei denen beide der untersuchten Sprachen nicht Englisch sind. Bei dieser Überblicksdarstellung berücksichtigt Huemer außerdem nicht nur Forschungsansätze aus dem europäischen Raum, sondern auch aus Asien und dem Nahen Osten.

Im Weiteren setzt sich Huemer kritisch mit der Sprachnutzung im Hochschulkontext auseinander. Sie hält fest, dass durch die Rolle des Englischen als Lingua franca andere Sprachen und die daran geknüpften kulturellen Diskurse marginalisiert werden. Gleichzeitig werden dadurch nicht nur der Hochschulkontext und damit die Lehre und wissenschaftliche Karrieren beeinflusst, sondern ebenso der wissenschaftliche Publikationsbetrieb.

Im ersten Beitrag des Teils, didaktische Herangehensweisen, gibt Donahue zunächst einen Überblick über die Forschung der letzten 50 Jahre zum Thema >Schreiben< aus unterschiedlichen kulturellen Kontexten. Ziel ist keine neue Schreibpädagogik, sondern die Forderung nach einer Neubetrachtung und Neubewertung bestehender Vorstellungen zum wissenschaftlichen Schreiben und der daraus resultierenden Pädagogik. Anhand der Konzepte >Translingualität<, >Multikompetenz< und >Heteroglossie< macht die Autorin deutlich, dass im heutigen Kontext die (Schreib-)Anforderungen an Studierende wechselnd sind und ihr Können daher flexibel und anpassbar sein muss. Donahue hält fest, dass wissenschaftliches Schreiben besonders eine Übertragbarkeit, also Wiederverwendung und Anpassung des Sprach- und Diskurswissens, an unterschiedliche Kontexte erfordert. 
Im zweiten, praktisch ausgerichteten Beitrag stellen Kuitunen und Carolan ein konkretes Konzept für die fachinterne Vermittlung des wissenschaftlichen Schreibens am Beispiel des Fachs Geschichte vor. Dabei gehen die AutorInnen zunächst auf die besondere Sprachsituation in Finnland ein, die aus zwei Amtssprachen (Finnisch und Schwedisch) resultiert. Kuitunen und Carolan präsentieren in ihrem Beitrag das Programm language and communication studies, das Studierende in die mündlichen und schriftlichen Praktiken des wissenschaftlichen Schreibens einführt und auf recht abstrakte Lernziele ausgerichtet ist. Darüber hinaus wird das Feedback Studierender $\mathrm{zu}$ diesem Programm vorgestellt.

Gegenstand des dritten Beitrags sind Heckenausdrücke und ihre Bedeutung für die wissenschaftliche Kommunikation. Vold vergleicht Studien zu Heckenausdrücken und stellt erhebliche kulturelle Unterschiede in ihrer Verwendung fest. Daraus resultierend fragt Vold nach einem passenden didaktischen Ansatz für wissenschaftliche Schreibkurse zur Vermittlung von Heckenausdrücken. Sie wägt die Bildung gruppenspezifischer Vermittlungsmethoden gegen einen plurilingualen Ansatz ab und spricht sich für Letzteren aus.

Im ersten Beitrag des zweiten Teils, sprachliche Merkmale, stellt Gardner das Korpus British Academic Written English (BAWE) vor. Im BAWEKorpus sind Texte Studierender aus 300 Studiengängen enthalten, die sowohl unterschiedliche Textsorten (z.B. Essays oder Forschungsberichte) umfassen als auch aus unterschiedlichen Studienphasen (erstes bis viertes Stu- dienjahr) stammen. Gardner benennt Studien, die dieses Korpus für sprachvergleichende Analysen verwendet haben, und präsentiert insbesondere mehrere Untersuchungen, die BAWE für einen Sprachvergleich des wissenschaftlichen Schreibens von chinesischen und englischen SchreiberInnen nutzen. Der Beitrag schließt mit Hinweisen auf das Forschungspotential des Korpus. Zudem werden die Befunde des Sprachvergleichs als Liste von Empfehlungen für die Didaktik des wissenschaftlichen Schreibens zusammengefasst.

Thielmann untersucht im fünften Beitrag die grammatische Zusammensetzung des Passivs im Englischen und Deutschen ausgehend davon, dass diese Form in wissenschaftlichen Texten primär als Möglichkeit der Agensunterdrückung betrachtet wird. Er macht deutlich, dass unser heutiges Verständnis einen sehr offenen Passivbegriff beinhaltet. Für die weitere Analyse begrenzt Thielmann das Passiv auf die entsprechenden Komponenten to be + past participle und sein bzw. werden + Partizip II und zeigt auf, dass das Passiv ebenso zur thematischen Strukturierung wie zur Leserführung genutzt werden kann. Darüber hinaus weist er darauf hin, dass zwischen dem deutschen und dem englischen Passiv durchaus ein Unterschied sowohl grammatischer als auch funktionaler Art besteht.

Im sechsten Beitrag ist der $>\mathrm{Li}$ teraturüberblick $<$ als Teiltext wissenschaftlicher Texte Gegenstand der Untersuchung. Hierfür haben Deroey, Huemer und Lejot zwölf Abschlussarbeiten in den Sprachen Deutsch, Englisch und Französisch von Masterstudierenden der Universität Luxem- 
burg analysiert. Diese Untersuchung ergab drei Elemente (Bericht, Diskussion und Textorganisation), die abhängig von der Sprache mit unterschiedlicher Häufigkeit auftraten. Ausgehend von den Befunden zeigen die Autorinnen Möglichkeiten eines mehrsprachigen Schreibkurses auf und benennen dessen Vorteile.

Eine Besonderheit dieses Beitrags besteht darin, dass alle Überschriften zwar auf Englisch, zwei Drittel des Textes jedoch auf Französisch und Deutsch geschrieben sind. Der im Vorwort erwähnte »kulturell und sprachlich hybrid[e] Mikrokosmos« (11), die Universität Luxemburg, wird in diesem Beitrag lebhaft veranschaulicht.

Dengscherz stellt im siebten Beitrag des Sammelbands vor, wie Sprachrepertoires und Sprachbiografien das Schreiben beeinflussen und wie im Schreibprozess mehrere Sprachen Anwendung finden können. Die Daten stammen aus dem Projekt PROSIMS (»Strategien und Routinen für professionelles Schreiben in mehreren Sprachen«) der Universität Wien zur Entwicklung einer Theorie mehrsprachigen Schreibens.

In der Analyse unterscheidet Dengscherz zwischen heuristischen und rhetorischen Anforderungen an das Schreiben. Anhand von zwei Fallbeispielen legt sie offen, dass SchreiberInnen individuell unterschiedliche Strategien nutzen, um ihre verschiedenen Sprachen einzubeziehen. Sie hält jedoch trotzdem einige übereinstimmende Tendenzen fest. So werden mehrere Sprachen beim inhaltsbezogenen Nachdenken (heuristische Anforderungen) genutzt, während das Formulieren hauptsächlich in der Zielsprache des Textes abläuft (rhetorische
Anforderungen). Im Formulierungsprozess können, so Dengscherz, mehrere Sprachen zur Wortschatzkompensation verwendet werden. Dengscherz hält fest, dass die Nutzung von Sprachen durch sowohl situative Faktoren als auch individuelle Zugänge beeinflusst wird.

Im achten Beitrag setzt sich Rheindorf mit der Autorpositionierung auseinander. Diese dient zum Aufbau von Autorität, Integrität und Glaubwürdigkeit des/der VerfasserIn selbst, so Rheindorf, und wird i.d.R. durch spezifische sprachliche Mittel mit feinen Abstufungen aufgebaut. Ausgehend von bestehenden Studien zeigt Rheindorf die Schwierigkeit der Autorpositionierung am Beispiel österreichischer AbsolventInnen mit Deutsch als L1 und Englisch als L2, die Schwierigkeiten bei der Autorpositionierung im Englischen aufweisen. Anhand von Daten aus insgesamt 52 Kursen zum wissenschaftlichen Schreiben zeigt Rheindorf mithilfe von Verben zur (Rede-)Wiedergabe die Notwendigkeit, Studierende für die einzelnen Positionsabstufungen zu sensibilisieren.

Giannoutsou untersucht im neunten Beitrag, welchen Einfluss Mobilität, der globale Austausch und das Englische als Lingua franca auf die Arbeit von WissenschaftlerInnen haben. Hierfür führt sie teilstrukturierte narrative Interviews mit 17 WissenschaftlerInnen der Universität Hamburg durch und hält fest, dass die Befragten einen starken Druck spüren, sich im Englischen zu profilieren. Außerdem bedeutet mehrsprachiges Schreiben für die Probanden vorrangig Schreiben in ihrer Muttersprache und im Englischen. Damit hat 
das Englische als Berufssprache (professional English) erheblichen Einfluss auf die Sprachenpolitik, die alltäglichen Abläufe und die Hierarchien der Hochschule und damit auf die Wissenschafts- und Lehrpraxis.

Der erste Beitrag des vierten Teils ist m.E. ein besonderer Text. Deroey fasst die Kernaussagen der Podiumsdiskussion zusammen, die im Rahmen des Symposiums 2016 stattfand. In der Verschriftlichung hält Deroey nicht nur die Aussagen selbst fest, sondern berücksichtigt auch, welche/ welcher TeilnehmerIn die Aussage gemacht hat. Im Rahmen der Podiumsdiskussion wurden hochaktuelle Themen besprochen: die Ausgangslage an den Hochschulen im Umgang mit Mehrsprachigkeit, das Wissen um mehrsprachige Schreibprozesse und die daraus resultierenden didaktischen Implikationen ebenso wie Forschungsmöglichkeiten. Der Beitrag schließt mit vier Kernaussagen zur akademischen Schreibforschung und Lehre.

Redder knüpft im elften Beitrag an die Podiumsdiskussion an. Sie geht darauf ein, dass die diverse Mehrsprachigkeit der AkteurInnen für die akademische Bildung ausgeschöpft werden sollte. Damit stellt Redder das Englische als Lingua franca in Frage und argumentiert für eine komparative und mehrsprachige Wissenschaftsbildung, die durch die Einrichtung eines »Zentrum[s] für die Komparatistik der Wissenschaftskommunikation« (261) entsprechende hochschulund wirtschaftspolitische Maßnahmen finden soll.

Der Band schließt mit einer $\mathrm{Zu}$ sammenfassung der Ergebnisse aller Beiträge. Lejot gliedert diesen Text in drei Teilabschnitte. Sie beschreibt zunächst beispielhaft einzelne Projekte, die Mehrsprachigkeit erforschen und sich mit der Vermittlung von Sprachen im Hochschulkontext beschäftigen. Anschließend bewertet Lejot die Beiträge des Bandes und stellt einerseits fest, dass wissenschaftliche Studien unterschiedlicher Sprachen mehr Gemeinsamkeiten als Unterschiede aufweisen und andererseits die Bedeutung von Nuancen zwischen den Sprachen von den linguistischen Studien des Bandes betont wird.

Weiter macht Lejot Vorschläge für weiterführende Forschung und gibt dabei einige positive Beispiele aus dem studentischen Tandemlernen. Der Beitrag schließt mit dem Hinweis, dass nicht nur ein Vergleich zwischen den Sprachen wichtig ist, sondern ebenso der interdisziplinäre Vergleich innerhalb einer Sprache berücksichtigt werden sollte.

Der Sammelband ist nicht nur inhaltlich gründlich redigiert. Auch äußerlich handelt es sich um ein wertig präsentiertes Buch. Die drei Sprachen des Titels sind jeweils unterschiedlich hervorgehoben. Dabei fällt das Englische durch Fettmarkierung besonders auf und knüpft so an eine inhaltliche Aussage an: die zentrale Stellung des Englischen in der Wissenschaft. Die Mehrsprachigkeit wird zudem durch einen bereits auf dem Buchcover im abgebildeten Vierfarbkugelschreiber verkörpert, der als Metapher für einen Menschen (der Kugelschreiber) mit unterschiedlichen Sprachkenntnissen (Farben) betrachtet werden kann. Ein kleines Detail, dass ebenfalls zum positiven Gesamteindruck beiträgt, sind die unterschiedlichen Perspektiven auf den Stift. Er ist aus insgesamt drei 
Blickwinkeln sichtbar, wenn man das Buch aufschlägt und den Einband von außen als Ganzes betrachtet.

Zusammenfassend lässt sich sagen, dass der Sammelband nicht nur theoretische Betrachtungen und praktische Ansätze verbindet, sondern auch die Bandbreite schreibwissenschaftlicher Forschung sprachenübergreifend abdeckt. Damit bietet er sowohl einen Einstieg in die Thematik des mehrsprachigen akademischen Schreibens als auch neue Gedanken und Ansätze für ForscherInnen des Felds. Das Versprechen des Einbands, »eine Bereicherung für das wissenschaftliche Schreiben in mehrsprachiger Umgebung « zu sein, wird eingelöst, wobei m.E. nicht nur die Beiträge eine Bereicherung sind, sondern auch der Gesamteindruck, der sich zum positiven Vorbild eines Sammelbands zusammensetzt, der ganzheitlich, durchdacht und mehrsprachig ist. Damit bildet das Buch einen gelungenen Auftakt für die vielversprechende, zukunftsweisende Reihe Schreibwissenschaft.

Anna Tilmans

\section{Walter Fähnders: Projekt Avantgarde. Avantgardebegriff und avantgardistischer Künstler, Manifeste und avantgardistische Arbeit}

Bielefeld: Aisthesis 2019 - ISBN 978-3-8498-1310-9 - 19,80€

Seit Beginn des 21. Jahrhunderts jähren sich die Jubiläen der Avantgarde. Walter Fähnders hat nun einen Projekt Avantgarde betitelten Band vorgelegt, der nicht nur seine bisherigen Forschungen miteinander vernetzt, sondern zudem eine zuverlässige Basis für die Arbeit mit und an der historischen Avantgarde liefert.

Eine Reihe von Jubiläen haben in den letzten Jahren die Erinnerung an die europäischen Avantgarden aktualisiert, so z.B. das Dada-Jubiläum 2016. ${ }^{1}$ Doch schon zuvor wurde im Centenaire $^{2}$ europaweit an den Ersten Welt-

1 | Vgl. online unter: http://www.dada100 zuerich2016.ch/ [Stand: 1.4.2020].

2 | Unter www.centenaire.org informiert die République Française über die Maßnahmen der Erinnerung und Erforschung des Ersten Weltkriegs sowie die damit ver- krieg erinnert. Keine konzertierte Erinnerung gab es in Deutschland, nur in Nordrhein-Westfalen stellte das umfangreiche Verbundprojekt $\gg 1914-$ Mitten in Europa « Fragen an die Geschichte des Ersten Weltkriegs. Die Auftaktkonferenz dieses Projektes im September 2013 war mit Aggression und Avantgarde betitelt. Das Begriffspaar lässt natürlich zunächst an die Anfänge der Begriffsgeschichte im Militärischen denken - Avantgarde als

bundenen Vermittlungskonzepte 2014. Wikipedia verzeichnet einen Eintrag über die europäischen Ereignisse zum Centenaire, allerdings ohne das deutschlandweit einzigartige Verbundprojekt "1914 - Mitten in Europa" des Landschaftsverbands Rheinland, vgl. online unter: https:// en.wikipedia.org/wiki/First_World_War_ centenary [Stand: 1.4.2020]. 